\section{Immune checkpoint inhibitor rechallenge in patients with immune-related myositis}

Therapeutic management of many cancers has been revolutionised by the development of immune checkpoint inhibitors (ICI) targeting antiprogrammed death 1 (PD-1)/ligand 1 (PDL1) and anticytotoxic T-lymphocyte antigen 4 leading to durable responses. ${ }^{1}$ ICIs however can induce several immune-related adverse events (irAE) including musculoskeletal irAEs. ${ }^{2}$ Among them, ICI-related myositis can be severe and sometimes life threatening. ${ }^{3}{ }^{4}$ The current management includes permanent discontinuation of ICIs and steroid treatment. To date, very little is known about the risk of irAE recurrence in case of ICI rechallenge, ${ }^{56}$ especially in myositis for which no case of rechallenge has yet been reported. Through two cases, we report the safety of resuming anti-PD-1/PDL1 in patients who experienced severe ICI-related myositis.

An 87-year-old patient with metastatic Merkel cell carcinoma (MCC) received avelumab as first-line treatment. After three infusions, he developed slight head dropped syndrome with increased creatine kinase (CK) level up to 3.5 times the upper limit normal (ULN) range. Electromyography showed myogenic syndrome (left trapezius and right sternocleidomastoid muscles) and ${ }^{18}$ F-fluorodeoxyglucose-positron emission tomography revealed significant hypermetabolism of axial muscles (table 1). Myositis-specific autoantibodies were negative. Myocarditis was ruled out. At that time the radiologic evaluation showed a partial tumour response. Avelumab was stopped and the patient received prednisone (tapering from $0.5 \mathrm{mg} / \mathrm{kg}$ ) during 6 weeks, which allowed myositis remission, but MCC recurred 7 months later. Avelumab was resumed and prednisone was preventively given during 3 months, starting at $20 \mathrm{mg} /$ day. With a 9 -month follow-up, no irAE, including myositis, occurred and MCC returned in partial response.

A 61-year-old patient with metastatic melanoma developed ptosis, diplopia, dysphagia and muscle weakness 3 weeks after first infusion of ipilimumab combined with nivolumab as firstline treatment. CK levels raised up to $40 \mathrm{ULN}$. Electromyography showed myogenic pattern of the trapezius, without decrement. Muscular biopsy with focal necrosis/regeneration lesions, HLA-1 and C5b9 positive sarcoplasmic staining of the suffering myofibres and $\mathrm{T}$ cell infiltrates confirmed the myositis.

Table 1 Patient characteristics

\begin{tabular}{|c|c|c|c|}
\hline & & Patient 1 & Patient 2 \\
\hline \multirow[t]{2}{*}{ Cancer history } & Stage IV cancer & Merkel cell carcinoma & Melanoma \\
\hline & $\mathrm{ICI}$ & Avelumab $10 \mathrm{mg} / \mathrm{kg} / 2$ weeks, 3 infusions & $\begin{array}{l}\text { Ipilimumab } 3 \mathrm{mg} / \mathrm{kg}+\text { nivolumab } 1 \mathrm{mg} / \mathrm{kg}, 1 \\
\text { infusion }\end{array}$ \\
\hline \multirow[t]{5}{*}{$\mathrm{ICl}$-related myositis } & Onset of symptoms & Week 6 & Week 3 \\
\hline & Clinical symptoms & Dropped head syndrome & Myalgia, muscle weakness \\
\hline & & Neck pain & Ptosis, diplopia, dysphagia \\
\hline & & Fatigue & Fatigue \\
\hline & Maximum & $\times 3.5$ ULN & $\times 40$ ULN \\
\hline
\end{tabular}

Creatine kinase

Electromyography

FDG-PET

Muscular biopsy

Cardiac examination

ECG

High-sensitive troponin-T (<14 ng/L)

Echocardiography

Cardiac MRI

Cardiac FDG PET

Treatment of irAE

\begin{tabular}{|c|c|}
\hline \multirow[t]{6}{*}{ Rechallenge } & Response to initial treatment with $\mathrm{ICI}$ \\
\hline & Time from first $\mathrm{ICI}$ treatment to $\mathrm{ICI}$ rechallenge \\
\hline & $\mathrm{ICl}$ \\
\hline & Associated treatment \\
\hline & irAE \\
\hline & $\mathrm{ICl}$-myositis \\
\hline
\end{tabular}

Tumour response
Myogenic syndrome: Left trapezius and right sternocleidomastoid muscle. No decrement.

Significant hypermetabolism of trapezius, erector muscles Slight diffuse muscular hypermetabolism of the spine, pilar muscle of the diaphragm

Contraindication (anticoagulant treatment for atrial fibrillation)

Permanent atrial fibrillation ( $>5$ years)

475

Normal

Contraindication (pacemaker)

No myocarditis

Oral prednisone $0.5 \mathrm{mg} / \mathrm{kg} / \mathrm{day}$, tapered and withdrawn within 6 weeks

\section{Partial response}

7 months

Avelumab $10 \mathrm{mg} / \mathrm{kg} / 2$ weeks, 20 infusions

Prednisone $20 \mathrm{mg} /$ day, tapered and withdrawn within 3 months

None

No recurrence of myositis with no symptoms, normal CK level, normal electromyography and absence of muscle hypermetabolism on TEP

Ongoing partial response after 9 months lesions, HLA-I and C5b9 positive staining, presence of $\mathrm{T}$ cell and macrophage inflammatory cells

Normal

298

Normal

\section{No myocarditis}

Methylprednisolone $1 \mathrm{~g} /$ day $\times 3$ days, followed by oral prednisone $1 \mathrm{mg} / \mathrm{kg}$, tapered and withdrawn within 9 weeks

Progressive disease

8 months

Pembrolizumab 2 mg/kg/3 weeks, 2 infusions

None

No recurrence of myositis without any symptoms, normal CK level

Death due to progressive disease at week 6

CK, creatine kinase; FDG-PET, fluorodeoxyglucose-positron emission tomography; HLA, human leucocyte antigen; ICl, immune checkpoint inhibitor; irAE, immune-related adverse event; ULN, upper limit normal. 
Neither myositis-specific nor myasthenia gravis autoantibodies were detected. ICIs were stopped and three pulses of methylprednisolone followed by tapering doses of prednisone were given leading to complete remission within 8 weeks. Because of the lack of efficacy of the single infusion of ICI combination followed by three infusions of dacarbazine, pembrolizumab was introduced 8 months after the myositis episode. The patient presented no irAEs or myositis (table 1) but died due to melanoma progression.

Despite the risk of recurrent irAEs, rechallenging ICIs after discontinuation due to previous irAEs remains critical, when considering their potential benefits in terms of survival. ${ }^{5}$ These two cases suggest that resuming ICIs can be safe in patients displaying persistent remission of ICI-related myositis.

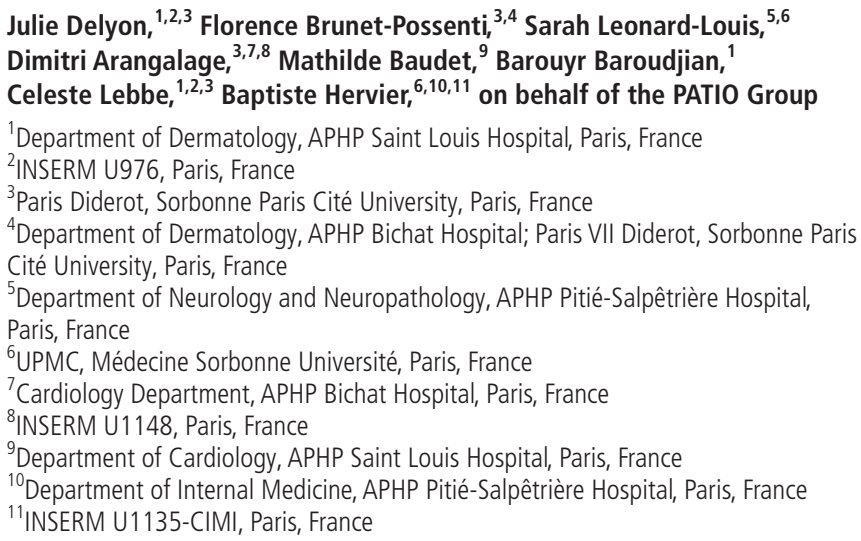

Correspondence to Dr Julie Delyon, Dermatology, APHP Saint Louis Hospital, Paris 75475, France; julie.delyon@aphp.fr

Contributors JD and FBP analysed the data and drafted the manuscript for intellectual content. SLL, DA, MB and BB had a major role in the acquisition of data. $\mathrm{CL}$ and $\mathrm{BH}$ interpreted the data and revised the manuscript for intellectual content. All authors approved the submitted version of the manuscript.
Funding The authors have not declared a specific grant for this research from any funding agency in the public, commercial or not-for-profit sectors.

Competing interests $B H, D A, J D, F B P, M B$ and SLL had nothing to declare. $B B$ received payments for lectures/boards and travel accommodations from BMS and MSD. CL received research grants or honoraria from Roche, BMS, MSD, GSK, Novartis and Amgen.

Patient consent Obtained.

Ethics approval Local ethics committee.

Provenance and peer review Not commissioned; internally peer reviewed.

(c) Author(s) (or their employer(s)) 2019. No commercial re-use. See rights and permissions. Published by BMJ.

\section{Check for updates}

To cite Delyon J, Brunet-Possenti F, Leonard-Louis S, et al. Ann Rheum Dis 2019;78:e129.

Received 24 August 2018

Accepted 30 August 2018

Published Online First 21 September 2018

Ann Rheum Dis 2019:78:e129. doi:10.1136/annrheumdis-2018-214336

\section{REFERENCES}

1 Wolchok JD, Chiarion-Sileni V, Gonzalez R, et al. Overall survival with combined nivolumab and ipilimumab in advanced melanoma. N Eng/ J Med 2017;377:1345-56.

2 Kostine M, Rouxel L, Barnetche T, et al. Rheumatic disorders associated with immune checkpoint inhibitors in patients with cancer-clinical aspects and relationship with tumour response: a single-centre prospective cohort study. Ann Rheum Dis 2018;77:393-8.

3 Touat M, Maisonobe T, Knauss S, et al. Immune checkpoint inhibitor-related myositis and myocarditis in patients with cancer. Neurology 2018;91:e985-94.

4 Eggermont AMM, Blank CU, Mandala M, et al. Adjuvant pembrolizumab versus placebo in resected stage III melanoma. N Engl J Med 2018;378:1789-801.

5 Pollack MH, Betof A, Dearden H, et al. Safety of resuming anti-PD-1 in patients with immune-related adverse events (irAEs) during combined anti-CTLA-4 and anti-PD1 in metastatic melanoma. Ann Oncol 2018;29:250-5.

6 Menzies AM, Johnson DB, Ramanujam S, et al. Anti-PD-1 therapy in patients with advanced melanoma and preexisting autoimmune disorders or major toxicity with ipilimumab. Ann Oncol 2017;28:368-76. 